\title{
Analyzing the Impact of COVID-19 Control Policies on Campus Occupancy and Mobility via WiFi Sensing
}

\author{
CAMELLIA ZAKARIA, AMEE TRIVEDI, and EMMANUEL CECCHET, University of \\ Massachusetts Amherst, USA \\ MICHAEL CHEE, Duke-NUS Medical School, Singapore \\ PRASHANT SHENOY, University of Massachusetts Amherst, USA \\ RAJESH BALAN, Singapore Management University, Singapore
}

\begin{abstract}
Mobile sensing has played a key role in providing digital solutions to aid with COVID-19 containment policies, primarily to automate contact tracing and social distancing measures. As more and more countries reopen from lockdowns, there remains a pressing need to minimize crowd movements and interactions, particularly in enclosed spaces. Many COVID-19 technology solutions leverage positioning systems, generally using Bluetooth and GPS, and can theoretically be adapted to monitor safety compliance within dedicated environments. However, they may not be the ideal modalities for indoor positioning. This article conjectures that analyzing user occupancy and mobility via deployed WiFi infrastructure can help institutions monitor and maintain safety compliance according to the public health guidelines. Using smartphones as a proxy for user location, our analysis demonstrates how coarse-grained WiFi data can sufficiently reflect the indoor occupancy spectrum when different COVID-19 policies were enacted. Our work analyzes staff and students' mobility data from three university campuses. Two of these campuses are in Singapore, and the third is in the Northeastern United States. Our results show that online learning, split-team, and other space management policies effectively lower occupancy. However, they do not change the mobility for individuals transitioning between spaces. We demonstrate how this data source can be a practical application for institutional crowd control and discuss the implications of our findings for policymaking.
\end{abstract}

CCS Concepts: • Human-centered computing $\rightarrow$ Ubiquitous and mobile computing; • Applied computing $\rightarrow$ Health informatics;

Additional Key Words and Phrases: COVID-19, occupancy, mobility, campus, WiFi, analysis, large-scale

\section{ACM Reference format:}

Camellia Zakaria, Amee Trivedi, Emmanuel Cecchet, Michael Chee, Prashant Shenoy, and Rajesh Balan. 2022. Analyzing the Impact of COVID-19 Control Policies on Campus Occupancy and Mobility via WiFi Sensing. ACM Trans. Spatial Algorithms Syst. 8, 3, Article 22 (September 2022), 26 pages.

https://doi.org/10.1145/3516524

This research is supported by NSF grants 1763834, 1836752, 2021693, 2020888, 2105494 and the Singapore Management University Lee Kong Chian Fellowship.

Authors' addresses: C. Zakaria, A. Trivedi, E. Cecchet, and P. Shenoy, Manning College of Information and Computer Sciences University of Massachusetts Amherst 140 Governors Dr, Amherst, MA 01002; emails: \{nurcamellia, amee, cecchet, shenoy\}@cs.umass.edu; M. Chee, Yong Loo Lin School of Medicine National University of Singapore 10 Medical Dr, Singapore 117597; email: michael.chee@nus.edu.sg; R. Balan, School of Computing and Information Systems 1 Singapore Management University 80 Stamford Road, Singapore 178902; email: rajesh@smu.edu.sg.

Permission to make digital or hard copies of all or part of this work for personal or classroom use is granted without fee provided that copies are not made or distributed for profit or commercial advantage and that copies bear this notice and the full citation on the first page. Copyrights for components of this work owned by others than ACM must be honored. Abstracting with credit is permitted. To copy otherwise, or republish, to post on servers or to redistribute to lists, requires prior specific permission and/or a fee. Request permissions from permissions@acm.org.

(C) 2022 Association for Computing Machinery.

2374-0353/2022/09-ART22 \$15.00

https://doi.org/10.1145/3516524

ACM Transactions on Spatial Algorithms and Systems, Vol. 8, No. 3, Article 22. Publication date: September 2022. 


\section{INTRODUCTION}

Mobile sensing is increasingly employed to provide community support in security and safety $[18,34]$ and, very quickly, has proven helpful in the recent COVID-19 global pandemic. For example, countries such as Singapore and South Korea promptly adopted mobile apps and sensors for various pandemic responses, including contact tracing and guiding social distancing policies $[16,17,46]$. As our understanding of COVID-19 constantly expands, data-driven analytics have paved the way to guide safe management measures by governments worldwide [15, 43]. Overall, these efforts have aided in revising health guidelines and even re-openings to crowds [13]. Unfortunately, monitoring the safety compliance of these policies can be a struggle for agencies, organizations, and institutions as they rapidly change with new findings.

Sensing location information is fundamental in digital solutions responding to COVID-19. For example, users' current location within communal spaces infers crowd density, allowing operations management to mobilize social distancing measures. Their location histories help identify places at risk of virus exposure in contact tracing procedures. Much research analyzing location data to combat COVID-19 has been conducted over this short term. Badr et al. analyzed mobile phone signals from cell towers in 25 U.S. counties and found that mobility patterns strongly correlate with the virus spread [4]. Positioning systems using Bluetooth and GPS immediately became pivotal in digital contact tracing apps to stop the viral spread [13]. Besides these mobile apps, crowd monitoring solutions (CMSs) are also relevant to monitor gathering in enclosed spaces [3, 25, 57]. Recent efforts utilizing CMS for COVID-19 include video images [5, 29, 48] and Bluetooth [61]. Fundamentally, these solutions can procedurally track occupants' digital footprints of their physical whereabouts. Bluetooth, drones, or thermal imaging cameras are less privacy invasive than typical video surveillance. However, institutions may lack operational readiness, requiring additional device deployment in dedicated environments. A feasible solution is leveraging the existing WiFi network typically available to occupants in educational institutions [53,60]. These works proposed inference methods to identify at-risk occupants or superspreader events based on their associations with specific access points (APs) and contact graphs to reveal potential directional interactions between individuals. The effectiveness of these techniques was demonstrated through simulated examples.

In the same vein, our work seeks to investigate if location data that is passively sensed from existing WiFi infrastructure can, in fact, show the real-world effects of various COVID-19 policies on institutions when the pandemic broke out. Our goal is to show that coarse-grained WiFi data can sufficiently reflect the spectrum in crowd change when different control policies are implemented over time within an institution. Monitoring crowd change at three-level granularity (i.e., area, floor, and building) can contribute to the institutions' ability to report on actual space utilization in addition to specific details, such as total building capacity, buildings' floor-by-floor, and area metrics. Besides being alerted of densely populated parts of the building, monitoring building occupancy and mobility is critical in informing the maximum range of occupancy and assessing exposure and vulnerabilities from occupants' mobility across shared open spaces. We present findings from analyzing anonymized and aggregated students' and staff's location data through collecting WiFi logs directly from deployed campus WiFi infrastructures across three universities. ${ }^{1}$ Two of these campuses are in Singapore, and one is in the northeastern part of the United States. We employ two key measures: (1) occupancy, the number of users represented by unique smartphone device counts in each building, and (2) mobility, the order of buildings visited by the user.

This analysis comprises data during the initial phases of COVID-19 for all universities in both countries. Our results show occupancy and mobility changing with different levels of restrictions.

${ }^{1}$ Privacy and ethical considerations for our work are defined in Section 2.4. 
For example, we observed a reduction in occupancy when online learning was gradually introduced to some undergraduate classes. However, for on-campus staff and students, their mobility rate remained high. In contrast, a significant decrease in mobility rate is observed during the remote learning period. It is important to emphasize that this work neither solves a prediction problem nor proposes an alternative to digital contact tracing. Instead, our findings show the efficacy of WiFi sensing technique in regulating institutional compliance. The coarse-grained data analysis prevails as a data-driven approach to minimize congregation, moderate building occupancy, and crowd movement at an aggregated scale, preserving user privacy. Overall, this article makes the following contributions:

(1) It provides one of the first detailed looks at the effect of COVID-19-related policies on three campus populations across two different countries. Our results show how the occupancy and mobility metrics change across all three campuses as COVID-19 quarantine policies, of increasing severity, are enacted.

(2) It provides evidence that passive monitoring of indoor occupancy and mobility patterns using WiFi data could be useful to administrators in deciding the appropriate safe distancing measures for similar disease outbreaks.

\section{BACKGROUND AND MOTIVATION}

In what follows, we summarize prior work relevant to digital COVID-19 contact tracing and the practical use of WiFi sensing in the existing literature to monitor indoor occupancy and mobility for various applications.

\subsection{COVID-19 College Outbreak}

Close contact and congregations are discouraged during the pandemic since COVID-19 can spread from person to person through respiratory droplets or by breathing in contaminated air. Some studies have reported that the virus can remain airborne over longer periods, especially in indoor spaces where they may likely have reduced ventilation [8]. The virus poses a direct threat to highpopulation-density areas, particularly work and education environments. Now more than ever, precise indoor positioning capabilities are necessitated in these environments to model human mobility and identify areas at risk of disease spread.

One of the earliest virus outbreaks among college students was determined in March 2020, when 64 students were diagnosed positive with COVID-19 as they returned to the United States from their spring break [32]. This outbreak was effectively controlled with swift cooperation and compliance between the university and public health officials. However, by July 2020, it was reported that 6,600 COVID-19 cases linked to 270 colleges nationwide had begun to spread even before the fall semester-proving university campuses as highly vulnerable to the disease outbreak [51]. As universities scrambled for campus closure, they were simultaneously pressured to respond to new public health strategies and requirements [7], ranging from implementing restrictions on population movement to creating makeshift quarantine sites [1].

More recently, the New York Times reported on the success of major Singapore universities preventing the spread of COVID-19. Specifically, Singapore universities developed monitoring systems to help residents comply with zone restrictions and other safety measures [52]. Simultaneously in the United States, the Centers for Disease Control and Prevention (CDC) has provided several revisions on guidelines with which educational institutions must comply to reopen safely [7]. Among these operation plans are decreasing occupancy in areas with limited indoor ventilation, staggering use, and restricting occupancy rate in shared spaces. The complexity of adapting rapidly 
changing rules and monitoring institutional compliance offers unprecedented mobile sensing opportunities to aid universities in making more informed decisions.

\subsection{Digital Technologies for Monitoring COVID-19}

Since its outbreak, the public health response to COVID-19 has quickly tapped into digital solutions to support various use-cases from public awareness to management protocols for different enduser groups such as the general public, facility administrators, and case investigators, respectively. Fundamentally, these efforts rely on localization and tracking methods to reduce the virus spread. This is particularly important as different countries, states, and even organizations enact a slew of policies they believe are best for them [24].

Several COVID-19 trackers are available online, showing the number of infections per country $[6,11,55,56]$, the spread rate [49], and possible ways to exit lockdown across the world [58]. At present, many crowd surveillance solutions used to support COVID-19 safety compliance leverage video, footfall counter, and a combination of IoT sensors to measure crowd density and monitor crowd movement and the usage rate of facilities [22]. Real-time crowd density apps utilizing occupancy sensors, cameras, and ticket validations are also piloted in European cities to help commuters make well-informed decisions on the best travel routes and times [23].

Smartphone-based Location Sensing. One of the earliest analyses using mainly location data from the user's smartphone investigated the cluster of COVID-19 cases in an office block in South Korea [46]. Confirmed-case patients supplied their GPS information and were notified of the nearest screening center to get tested. Mobile phones quickly became key for exposure notifications and safety guidelines $[4,13,31]$. Simultaneously, Singapore mandated mobile check-ins at public spaces, for example, a shopping mall and every store within it, for residents to log their entries and exits using either a mobile phone or scanning their national identification card [16]. While SafeEntry helps reduce manual logging effort for businesses, these procedures may be cumbersome to customers. Other solutions include digital contact-tracing mobile apps. Using Bluetooth and GPS, these apps track users' digital footprints of their whereabouts. Fundamentally, institutions can leverage such applications to manage their safety compliance. However, the sensing modalities pose several challenges. First, institutions may lack operational readiness, requiring the deployment of Bluetooth beacon devices in environments. Second, GPS struggles with indoor positioning from receiving inaccurate satellite signals. A viable solution is leveraging WiFi networks that are already deployed in institutions and are the most widely used facility by occupants.

\subsection{Leveraging WiFi-based Location Sensing}

Low user compliance in Bluetooth smartphone sensing for COVID-19 has strongly motivated our research to understand the usefulness of WiFi sensing as a complementary modality for future contact tracing efforts.

2.3.1 Indoor Crowd Monitoring Systems. The research community has long contributed to accurate and sustainable CMSs. They include proposing the use of video surveillance [25, 45], RF [3], and Bluetooth devices [57]. Recent CMSs for COVID-19 include using drones [29], cameras [48], thermal imaging [5], and Bluetooth beacons [61]. Unfortunately, many challenges persist in achieving lasting real-world impact due to factors such as the cost of deployment and maintenance and cloud processing requirements.

WiFi sensing has lately revealed success in tracking indoor user mobility patterns for CMSs [14]. Given widespread WiFi availability, these solutions are to understand consumer buying behaviors at shopping malls [20], visitor analytics at airports and convention centers [27], and students' health and work performance from deriving behavioral routines and social interactions 
on campus $[47,59]$. Recent applications for COVID-19 include leveraging WiFi sensing to identify at-risk occupants or superspreader events based on their associations with specific APs [53] and directional interactions between individuals [60]. The effectiveness of these techniques, however, was demonstrated through simulated examples.

Fundamentally, solutions to manage crowds measure the flow rate of people from one location to another; however, the location granularity for these modalities differs. For example, Bluetooth contact tracing mobile apps or camera-based CMSs provide finer-grained inter-user distance trajectories. While WiFi sensing cannot provide such granularity (note: we discuss this as part of limitations in Section 8), picking up on nomadic movements between areas on a floor between buildings within a vicinity can produce digital footprints useful for identifying flagged paths of potential exposure within and across buildings. Through real-world examples, we show how simply monitoring indoor occupancy and mobility of occupants in three universities with WiFi can reflect the spectrum of crowd change when different COVID-19 policies were introduced.

2.3.2 Network-centric Sensing. Mobile phones have become ubiquitous in our daily lives, presenting many opportunities for behavioral sensing. Prior studies on using the "phone as a sensor" have gained new behavioral data [19, 44, 54]. For example, smartphone use is prevalent among university students, constituting the largest demographic of smartphone users [12]. At the same time, WiFi-based networks have achieved near-ubiquitous coverage in campus buildings and outdoor spaces, accommodating increasing student demands for learning and leisure activities [9]. The ubiquitous availability of "phone as a sensor" for user behavior and WiFi-based networks as the sensing medium provide an ideal technology platform for our work.

Mobile phone sensing comes in two forms: client based and network based. In client-based approaches, sensing is performed directly from the phone. Such sensing requires either OS support or a client-side mobile app (running continuously in the background) to perform sensing measurements. GPS is an example of a client-side sensing method using the OS (and GPS chip) to localize the phone. Bluetooth operates in similar ways and has been used for proximity sensing in digital contact tracing tools during the COVID-19 pandemic. On the other hand, network-based phone sensing involves using the wireless network for sensing measurements. Many of these approaches are passive-which means they do not require active user involvement and can be performed via passive observations of the device. Network approaches have been used for indoor positioning systems (IPS) where multiple WiFi APs within range of the phone can "triangulate" the phone's location [26].

As discussed in Section 3.2, the ability to use WiFi as a network-side positioning system is a key technology enabler for our research. Specifically, when a (user's) smartphone connects to an access point, it follows that its current location is within the vicinity of a nearby AP. Since enterprise WiFi networks log each connection made by mobile devices to each of their APs, this log can be analyzed to infer crowd movement patterns across campus and the occupancy levels in different buildings (based on the number of smartphones connecting to each AP).

Table 1 summarizes the comparison between the Bluetooth/GPS-based sensing (client-centric), more commonly adopted by present solutions, and WiFi-based sensing (network-centric) technique. Our choice for sensing must be straightforward in overcoming the key adoption challenges faced by existing client-centric applications. Specifically, the sensing approach must:

(1) Take the Path of Least Resistance: Users need not install a dedicated mobile application or allow device permissions, such as what is required for Bluetooth or GPS-based sensing applications. Our technique can automatically discover and scan connected devices without explicit user interaction, making it much easier to deploy at scale. 
Table 1. Comparison between Two Forms of Mobile Sensing - Client vs. Network Centric

\begin{tabular}{|l|c|c|}
\hline Attributes & $\begin{array}{c}\text { Client Centric } \\
\text { (Bluetooth/GPS) }\end{array}$ & $\begin{array}{c}\text { Network Centric } \\
\text { (WiFi) }\end{array}$ \\
\hline User Input & App installation, allow permissions & None required \\
\hline Data Collection & Direct from user device & WiFi infrastructure \\
\hline Environment & Indoor and outdoor & Indoor and limited outdoor \\
\hline Sensing Frequency & App dependent & Limited to target environment \\
\hline Availability & BLE beacon instrumentation & WiFi network deployment \\
\hline Location Accuracy & Fine-grained & Coarse-grained, AP level \\
\hline Power Consumption & $\begin{array}{c}\text { High impact from apps } \\
\text { running in the background }\end{array}$ & $\begin{array}{c}\text { Minimal impact from usual } \\
\text { WiFi signal scanning }\end{array}$ \\
\hline Compatibility & App and device dependent & Device dependent \\
\hline Privacy/Security & $\begin{array}{c}\text { App dependent (types of data, } \\
\text { data collection practices) }\end{array}$ & $\begin{array}{c}\text { Only WiFi network events are } \\
\text { collected; identifiers are hashed }\end{array}$ \\
\hline
\end{tabular}

Highlighted rows indicate the key adoption challenges to overcome.

(2) Bypass User Device for Data Collection: In the same way that no user action is required above, no user device will be accessed for data collection. Instead, WiFi network events, such as "syslog" and "RTLS events," will be collected directly from the WiFi infrastructure. Many enterprise networks already commonly use these events for IT security and performance monitoring.

(3) Immediately Operate in Enclosed Areas: Operability, especially in indoor spaces, remains a challenge by GPS-based techniques due to inaccurate satellite signals. Bluetoothbased applications heavily depend on setting up BLE beacons within the vicinity. In contrast, wireless networks are increasingly popular and available, specifically in work and education environments. Sensing location, however, is limited to users within the target environment.

2.3.3 Challenge \#1: No WiFi Network. WiFi-based sensing is not without several challenges. A key assumption of our work is ubiquitous network coverage, in that WiFi coverage is present in all spaces where mobile sensing needs to be performed. With increasing and improving WiFi deployments on college campuses to meet student demands [9], it is reasonable to assume a nearuniversal WiFi coverage inside campus buildings. However, WiFi availability outdoors can vary with AP placements that are typically indoors. It is important to note that our goal is to monitor safety compliance following current public health guidelines, specifically in enclosed areas where COVID-19 spread will pose more risk to occupants.

2.3.4 Challenge \#2: Disconnected Users. The other key assumption is ubiquitous phone availability, in that every campus user has a smartphone with them at all times. This directly implies that our sensing mechanism will overlook a user with no phone. Nevertheless, much research has argued for a high percentage of smartphone users, particularly on college campuses [12].

Additionally, smartphone users utilizing cellular data will present as unconnected mobile devices in our approach. Despite widely available cellular data coverage, the WiFi network is still a preferred alternative provisioned for many online activities that demand low-latency and highbandwidth networks. These activities include video/music streaming and online gaming, which are enthusiastically engaged by students. A different source of unconnected mobile devices is visitors. While visitors of the university may not utilize the campus $\mathrm{WiFi}$, their device remains visible to the WiFi network. This is because both iOS and Android devices are set to scan for available WiFi networks periodically (even though no connection is established), albeit with an anonymous MAC address. Logs of unconnected devices make precise monitoring challenging, but these records can 
still present as coarse-grained information approximating visitor occupants. Nonetheless, a natural course of handling crises such as COVID-19 is clamping down on visitors (e.g., cancel open houses and conferences) to prevent the spread outside the population. Our work proceeds to manage the occupancy and mobility of university residents who must resume their day-to-day work/school practices. Note that moving forward, we refer to unconnected devices as "unassociated devices."

2.3.5 Challenge \#3: Coarse-grained Location. A final challenge is coarse-grained positioning because WiFi networks can only yield positions at the granularity of AP location. While precise location (and proximity) information is important for digital contact tracing, this requirement is not necessary to achieve our goal. Our focus is on analyzing aggregated occupancy trends and the overall mobility patterns across the campus environment, for which coarse-grained position information is more than adequate.

\subsection{Privacy and Ethical Considerations}

Despite the noble intentions of combating COVID-19, most digital solutions present the challenges of user privacy. WiFi-based sensing is no different, particularly since users' WiFi network data will automatically be collected and analyzed within the vicinity. Unlike most mobile sensing efforts for COVID-19, we hope to determine WiFi-based sensing as a feasible way for institutions to monitor and maintain compliance with current public health standards-this monitoring is accomplished at an aggregated scale and does not require identifying individual users occupying the facility. All identifiable information of users is anonymized and cannot be reverse-engineered. The main data source for our analysis, at present, is already being collected by network security administrators. Nonetheless, several privacy safeguards already exist to be put into practice. They are:

(1) No access to user device: Many users are likely unaware of the types and frequency of data being collected from their mobile devices. Indeed, no data will be transmitted directly from the user's device through WiFi sensing. Instead, our analysis will strictly utilize WiFi network data that is already collected by deployed wireless infrastructures. The frequency of data collection is constrained to the time users are within the vicinity. All identifiable information in the WiFi network data, particularly the MAC ID and username (if any), is anonymized using the SHA-2 hash.

(2) Established network security protocol: The National Institute of Standards and Technology (NIST) recommended that enterprise network security analysis is the best practice to build strong cybersecurity and protect an organization. Indeed, a variety of security logs, including WiFi network data, are already being used for auditing, supporting investigations, and identifying operational trends [28]. The need for this security protocol has led to laws and regulations compelling organizations to protect user privacy. Utilizing the same data source for COVID-19 safety compliance will follow the same established security standards.

(3) Emergency use authorization: It is important to note that our analysis, purposed for institutional safety compliance, is presented at an aggregated scale and does not include pinpointed behaviors. However, this information may be deemed critical to further a contact tracing investigation. In such an event, emergency disclosures will be handled by an authorized official. We believe a formal operational protocol to assess risk variations must be in place before any information disclosure. Only when an individual is identified as at risk can a public health case investigator obtain a de-anonymized copy of the information.

Data Ethics. All data used in this article was obtained directly from the campus infrastructure and bounded by the computing agreements agreed to by each WiFi user when they received their 
Table 2. Details of Each Campus Studied

\begin{tabular}{|c|c|c|c|c|}
\hline Campus & No. Buildings & No. Students & No. Staff & No. APs \\
\hline Singapore Management University - SMU & 7 & $\approx 9,000$ & $\approx 1,000$ & $\approx 800$ \\
National University of Singapore - NUS & $\approx 240$ & $\approx 40,000$ & $\approx 10,000$ & $\approx 13,000$ \\
University of Massachusetts Amherst - UMASS & $\approx 230$ & $\approx 30,000$ & $\approx 8,000$ & $\approx 5,500$ \\
\hline
\end{tabular}

WiFi credentials. These agreements allowed us to use their data for aggregate analysis as long as individual identifiers were not used. As such, every MAC address obtained from the WiFi infrastructure was hashed using a consistent one-way hash function, and no specific user details (e.g., login IDs) were used. All of the analysis used by this article focuses on large aggregates with no analysis of specific individuals performed.

\section{DATA COLLECTION AND METHODOLOGY}

Our approach is using WiFi data to infer occupancy and mobility. In what follows, we state our assumptions and pre-processing steps prior to clarifying the key measures.

\subsection{Data Collection}

Our datasets were collected directly from the production WiFi networks of three different university campuses. Two of these campuses (SMU and NUS) are in Singapore, and the last one (UMASS) is in the Northeastern portion of the United States. Two campuses are full-sized residential campuses with over 200 buildings each and $\approx 40 \mathrm{~K}$ to $50 \mathrm{~K}$ students and staff, while the last university is a small non-residential establishment with $\approx 10,000$ students and staff spread across seven buildings.

All three universities run Aruba-equipment-supplied WiFi networks, with one university also running a Cisco-equipment-supplied WiFi network in addition to an Aruba network. For the Aruba networks, we pulled the WiFi data directly from the infrastructure either using real-time location service (RTLS) APIs [2] or reading the system logs directly. For the Cisco network, we pulled WiFi data directly from the network using the Cisco Connected Devices (CMX) Location API v3 [10] (recently rebranded as Cisco DNA Spaces). In all cases, we obtained the following information: for all associated WiFi devices, the timestamp when the associated device was seen, the BSSID of the AP that saw the device, and the hashed client MAC address of the associated device. For two of the campuses, we can also obtain the same information (time seen, BSSID that saw the device, and hashed client MAC) for unassociated devices as well, i.e., devices with WiFi on that are just scanning.

We have associated device data from February 2020 onward for all three campuses, allowing us to clearly view campus occupancy and mobility patterns across campus before, during, and after COVID-19-related measures were implemented. Table 2 provides details of each of the three campuses as well as the data collected.

\subsection{Using WiFi Positioning System}

3.2.1 Key Assumptions. As described in Section 2.3, our analysis is based on the key assumption that most of our users frequently utilize campus WiFi on their own smartphones. A separate analysis on our US campus, UMASS, reported approximately $90 \%$ university residents carrying a smartphone with them at all times; specifically, our WiFi network events revealed 30,084 users comprising 24,791 student users and 5,293 staff/faculty. The remaining 10\% neither owned a smartphone nor chose to use the campus WiFi. Like COVID-19 digital contact tracing apps, a critical obstacle in enabling an effective crowd monitoring solution is mass user adoption. While our key assumption naturally disregards a small percentage of users, the approach can achieve a critical 


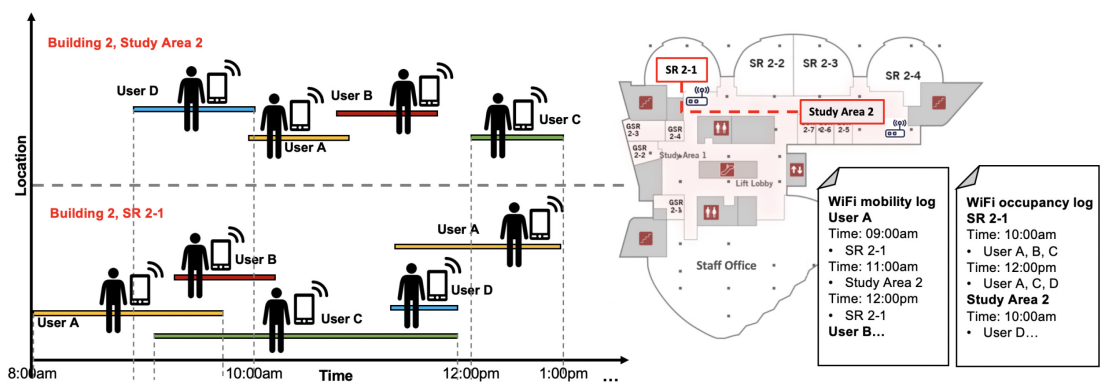

Fig. 1. Inferred occupancy and mobility based on smartphone WiFi connectivity to the nearest AP at floor level.

mass of data and ascertain some occupancy and mobility measures among smartphone users. In what follows, we describe how different mobile users can be tracked.

3.2.2 Pre-processing. We maintain similar pre-processing steps to extract information on human mobility for all campuses. For all WiFi enterprise networks deployed in the three universities, each AP on the network internally keeps a log of association, disassociation, authorization, and un-authorization activities with the devices that help us compute occupancy at various locations. An AP system log or "syslog" comprises a sequence of timestamped events. Each of these events follows the format:

hh:mm:ss <controller_name> <process_id > <event_subtype > <MAC_addr> <event_body>

For each event in syslog, an event_subtype representing the network event type is specified. This code can be recorded as six event types. They are association, disassociation, re-association, authentication, deauthentication, and drift events. Based on time (i.e., timestamp) and AP location, defined by controller_name and event_body, we can compute connection sessions per device across all APs [53]. For each device, we create a timestamp indexed sequence of sessions to acquire device trajectories. Combining these datasets helps us to characteristically produce user profiles (e.g., authenticated users as university residents) and activities (i.e., occupancy at a dedicated location or transitioning between locations). Note that MAC_addr is anonymized, as described in Section 2.4.

3.2.3 On-campus Deployment. We illustrate how network events (when the smartphone is connected to the campus WiFi) will produce time-based traces in Figure 1. A deployment of an enterprise WiFi network consists of many APs, optimally spread across buildings and floors.

When a user first utilizes the campus WiFi network on their own smartphone, authentication will occur (through their student ID and password), resulting in an authentication and deauthentication log message. Note, however, that student identification is hashed to preserve user privacy (see Section 2.4). Simultaneously, this action triggers the device to connect with the nearest AP, generating association log messages. The device continues to stay connected to the AP until the user moves; in this case, the connection switches to the next nearest AP to where the user is now situated. Accordingly, disassociation, re-association, and drift log messages will be generated when the user's device moves out of range or reconnects from "sleeping." Throughout the whole time, the user maintains the same network connection to the campus WiFi, only switching APs.

In Section 2.3.2, we discuss the challenges of accounting for unconnected devices, specifically devices that remain connected to their personal cellular network. This group of users will still be accounted for as long as their devices are scanning for a WiFi network (i.e., unassociated devices). It is important to note, however, that our main analysis only consists of associated devices, representing university staff and students. 


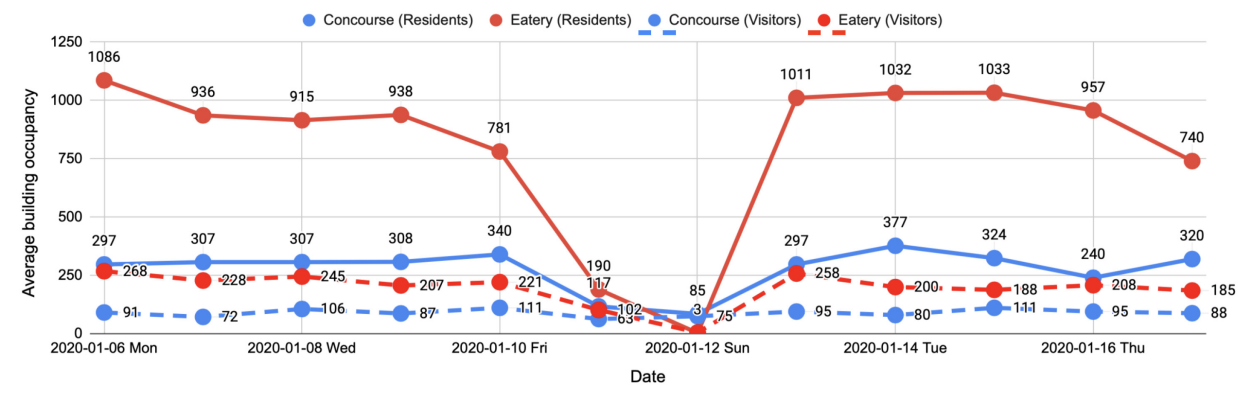

Fig. 2. Daily occupancy trends for two common areas, the concourse (blue) and the main eatery (red), based on associated devices (line) and unassociated devices (dashed line) on school days prior to the COVID-19 outbreak. Both areas are located on the same floor within a building at the SMU campus.

There are two other sources of error from utilizing this technique. First, a smartphone may maintain its existing connection to an AP due to good signal strength even though the user has moved. This error makes no impact on occupancy since we are counting on a per-floor/building basis, but will produce small errors on mobility. Second, a user may connect multiple devices (e.g., smartphone, laptop, wearable) to an AP at once. However, duplicated events can be removed [53], while device types can be further filtered to only collect one specific type if needed. This methodology has been validated by others $[20,33,53,59]$ and used for similar applications of mall analytics and queue management [26].

\subsection{Inferring Occupancy and Mobility Using WiFi Logs}

A campus network comprises several user types such as faculty, staff, students, on-campus student residents, and visitors. The syslog authentication event consists of login types, and thus helps differentiate a faculty/staff from a student. We further subdivide user groups based on the following rules. Students who spend more than 5 hours at an on-campus residential dormitory will be classified as on-campus student residents. Visitors are classified in several ways. First, records of anonymous MAC addresses are regarded as visitors (see Section 2.3.2). Second, users recorded with only a one-time login or devices with only several days of login over the course of the semester are most likely visitors. This heuristic is necessary to filter out users attending one-time events such as hack-a-thons, open houses, and conferences held on campus.

In Sections 4 and 5, respectively, our analyses will include reports of location occupancy at three-level granularity: area, floor, or building occupancy based on a collection of WiFi access points (i.e., AP location). For example, a large room such as a common dining hall can have more than one WiFi AP, while one AP can be at the intersection of different rooms. The coarse-grained information based on AP location amounts to inaccuracies in determining room-level information, especially if the room is small. Thus, an area can consist of a collection of small rooms (e.g., see Figure 1, GSR2-2, 2-3, 2-4 is regarded as a single area) or a singular large room (e.g., Figure 1, SR21). Accordingly, floor occupancy is the total of all areas on each floor, and building occupancy is occupancy on all floors in each building. We show in Section 4.2 the changes in occupancy rate over different COVID-19 phases, specifically for these areas.

3.3.1 Definition: Occupancy. We determine occupancy as the average number of people in a dedicated area of a single building floor, as shown in Figure 2. By taking the average occupancy at building level per day, our features can avoid the problem of missing data, common in longitudinal data collection procedures. The number of people is defined by unique device counts (smartphones) 

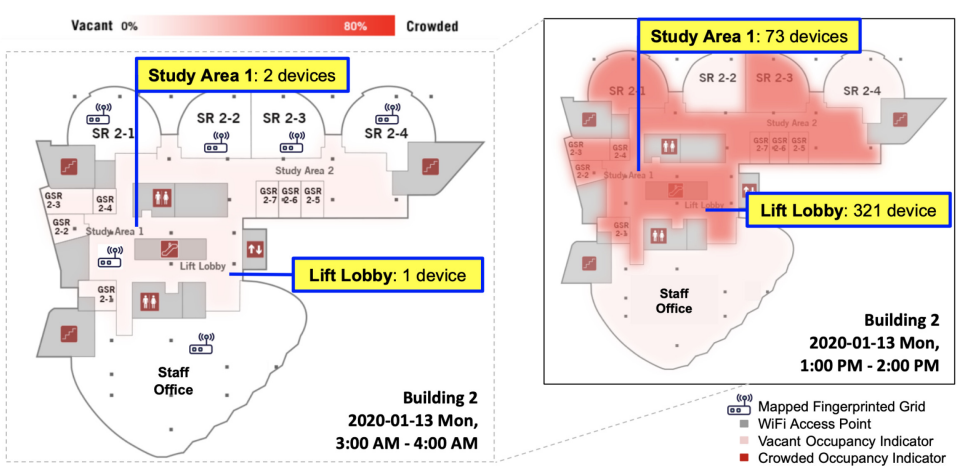

Fig. 3. Heat map of a floor in Building 2 of the $S M U$ campus. Left map shows the occupancy in the early morning, while right map shows occupancy during a regular school hour.

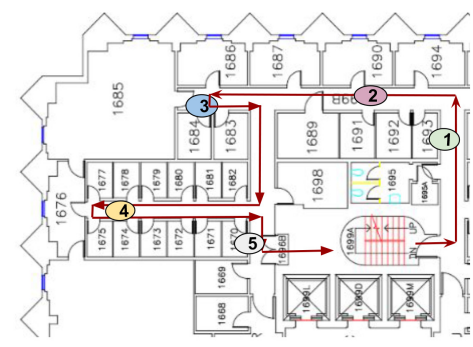

(a)

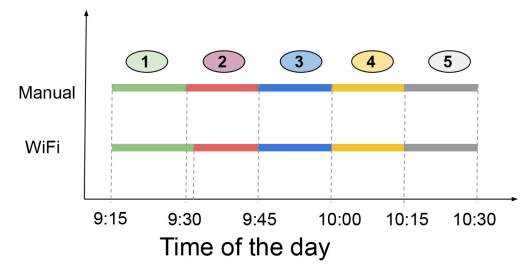

(b)

Fig. 4. (a) Floor map with AP locations within the UMASS campus. (b) Temporal lag between WiFi extracted trajectory and ground-truth manual log.

logged in our WiFi network records. The groups of people can also be known by identifying associated devices (for university residents) and unassociated devices (for visitors). For example, the SMUconcourse and main dining hall record approximately 100 to 200 visitors pre-COVID (January 6 to January 16,2020). This separation is necessary, especially since our universities are open campuses, providing pedestrian access to the public. Such information can aid institutions in identifying what percentage of occupants are university residents should they backtrack along the flagged path and areas vulnerable to exposure.

Examining our data from a different perspective, occupancy reveals cues about clusters on different areas of each floor building per hour. Figure 3 depicts a floor map in Building 2 at two time periods, one in the early morning and the other during school hours. By directly pulling a collection of network event logs from APs surrounding Study Area 1, we can determine two unique devices connected between 3:00 AM and 4:00 AM, indicating low building occupancy. In contrast, logs between 1:00 PM and 2:00 PM on a Monday show the expected high occupancy (e.g., 73 device connections at Study Area 1) throughout the hour.

3.3.2 Definition: Mobility. Mobility is defined as the average number of areas visited by a user per hour; once again, an area is defined by building level, measured for each floor comprising a collection of rooms on that ground. Figure 4 shows the floor map of a campus building in UMASS, comprising individual office units, classrooms, and open spaces. As a user moves around the floor through AP 1 to 5, indicated by the red arrow line in Figure 4(a), we observe changing network events from the device, generating association and disassociation events across different APs. The 


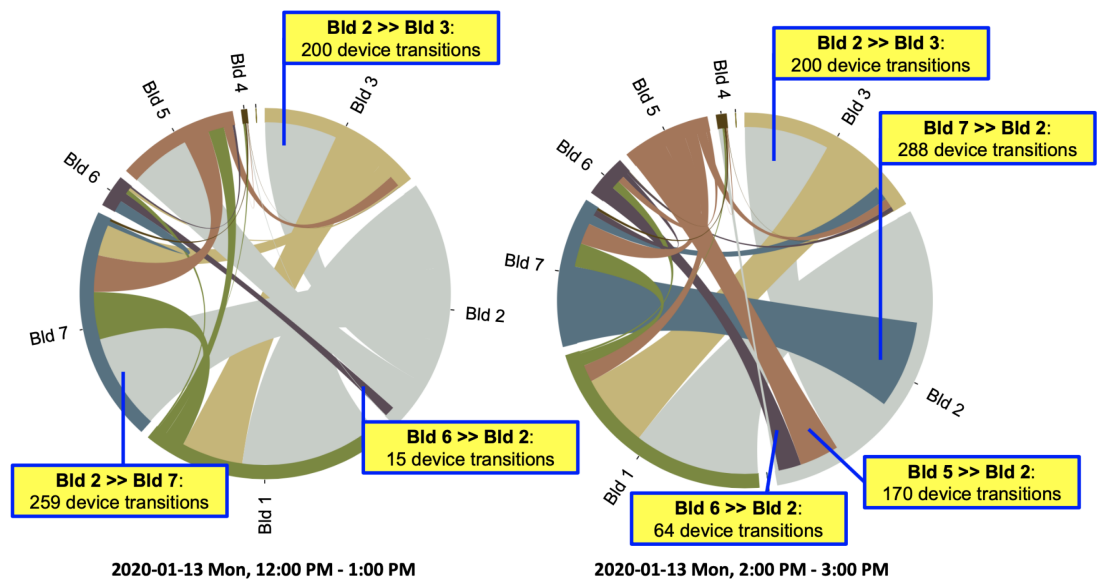

Fig. 5. Chord diagram representing the number of devices recorded to move from one building to another at different times of the day. The diagram displays all seven buildings within the SMU campus.

WiFi trajectory from one AP to another allows us to determine a user being "stationary" or "transitioning" between areas on the same floor. Figure 4(b) shows the temporal lag recorded for one user from switching to AP2 as he transitions. While temporal lags are likely to happen when APs are positioned close to one another, these inaccuracies will not affect our analysis in capturing building-level transitions.

In the context of COVID-19, one of the concerns is identifying possible transmission routes within and across the institution's buildings. Figure 5 comprises two chord diagrams representing the number of users recorded moving to and fro across seven different buildings within the $S M U$ vicinity. The left diagram illustrates much traveling into Building 7 from all other buildings during lunch hour, 12:00 PM to 1:00 PM. For example, 259 device transitions were made from Building 2 alone. In contrast, we observe more transitions distributed across buildings between 2:00 PM and 3:00 PM. For example, occupants recorded to be in Building 2 during that hour had visited Building 7 (288), 5 (170), and 6 (64 devices) before their transition. With some buildings being a single commonplace for occupants across campus to gather at different times of the day, this information can aid institutions to implement COVID-19 policies, for example, deploying social distancing support for regulating crowd control.

Conclusively, these statistics gathered from WiFi network logs can provide occupancy and mobility information, allowing us to monitor high-foot-traffic areas within the universities. In the next section, we investigate the adequacy of WiFi data in revealing the spectrum of changes in occupancy and mobility when the COVID-19 safety measures were enforced.

\section{MOBILITY AND OCCUPANCY ANALYSIS FOR SINGAPORE UNIVERSITIES}

We examine the changes in occupancy density and movements on campus to determine the effectiveness of various safety measures put in place at significant time points of COVID-19. These time points are summarized in Table 3.

\subsection{Overall Control Policy}

Singapore was first alerted of "severe pneumonia" cases in Wuhan city on January 2, 2020. From that point on, Singapore's Ministry of Health (MOH) has mandated a series of escalating control policies to prevent high infection rates of COVID-19 while minimizing significant disruptions 
Table 3. Five Phases of COVID-19-related Safety Measures Enacted in Singapore and at $S M U$ and NUS

\begin{tabular}{|c|c|c|}
\hline Singapore Phases & Date Started & Safety Measures \\
\hline \multirow{2}{*}{$\begin{array}{l}\text { Pre COVID-19 } \\
\left(P_{S} 0\right)\end{array}$} & Before & Awareness on personal hygiene \\
\hline & Feb. 19, 2020 & SMU moves some classes online \\
\hline \multirow[t]{7}{*}{ Phase $1\left(P_{S} 1\right)$} & Feb. 19, 2020 & National threat level raised to orange [40] \\
\hline & & $\begin{array}{l}\text { 14-day home quarantine enforcing if returning from } \\
\text { China [42] }\end{array}$ \\
\hline & & All core curriculum moved to online learning for NUS \\
\hline & & In-class mid-term assessment cancelled for NUS \\
\hline & & $\begin{array}{l}\text { Both } S M U \text { and NUS implement a 1-meter safe distancing } \\
\text { policy }\end{array}$ \\
\hline & & $S M U$ closes all sports facilities \\
\hline & & $\begin{array}{l}\text { Classes }>=50 \text { students moved to online learning for } S M U \\
\text { and NUS }\end{array}$ \\
\hline \multirow[t]{3}{*}{ Phase $2\left(P_{S} 2\right)$} & Mar. 22, 2020 & All travel cancelled unless mandatory \\
\hline & & $\begin{array}{l}\text { All visitors to Singapore issued 14-day Stay Home or- } \\
\text { der [21] }\end{array}$ \\
\hline & & $\begin{array}{l}\text { SMU enforcing A/B shifts where all students \& staff } \\
\text { must alternate being offsite every other week }\end{array}$ \\
\hline \multirow[t]{5}{*}{ Phase $3\left(P_{S} 3\right)$} & Apr. 3, 2020 & Full shift to online learning for all schools at all levels [41] \\
\hline & & All exams moved online at $S M U$ and NUS \\
\hline & & Pass/Fail option offered to students at SMU and NUS \\
\hline & & $S M U$ only allowing key personnel on campus \\
\hline & & NUS allowing most employers to work from home \\
\hline \multirow{5}{*}{$\begin{array}{l}\text { Phase } 4 \\
\left(P_{S} 4, \text { ongoing }\right)\end{array}$} & Apr. 7, 2020 & Full country-wide stay-at-home quarantine [37] \\
\hline & & Nobody allowed on campus for $S M U$; all buildings closed \\
\hline & & Only approved students allowed to stay in dorms at NUS \\
\hline & & Approved students allowed to travel to NUS to study \\
\hline & & Measures extended until June 2020 [39] \\
\hline
\end{tabular}

to the daily routines of its residents. The first case of COVID-19 in Singapore was reported on January 22, and more cases started appearing over the next few days. As shown in the subsequent phases $\left(P_{S} 1\right.$ to $P_{S} 4$, lockdown $\left.{ }^{2}\right)$, Singapore's $\mathrm{MOH}$ took increasingly strong measures to contain the spread of infection. These measures included mandatory stay-at-home quarantine orders for visitors, moving all academic programs online, and enforcing country-wide stay-at-home orders. Additionally, numerous facilities across Singapore were re-purposed as quarantine centers. For this analysis, several dormitory blocks at the NUS campus were re-purposed for this use in early May 2020 [50].

\subsection{Impact of Different Policies on Occupancy}

We first computed the drop in campus occupancy in Singapore as each phase of COVID-19-related policies were enacted. Figure 6 plots the unique device counts for one building within the Singapore universities, $S M U$ and NUS, over the COVID-19 phases, $P_{S} 0$ to $P_{S} 4$. We observed that there was a more than $90 \%$ percentage drop from phase $P_{S} 0$ to $P_{S} 3$ and beyond for $S M U$ when the university implemented an almost full work-from-home policy $\left(P_{S} 3\right)$ followed by the nationwide lockdown $\left(P_{S} 4\right)$. Despite taking the same set of measures, NUS was successful in reducing occupancy to only $75 \%$ at $P_{S} 3$ and $98 \%$ by the lockdown. For $S M U$, the drop was almost $100 \%$ by Phase $4\left(P_{S} 4\right)$ as nobody, except for security staff, was allowed onto campus, whereas NUS still allowed a few thousand students to stay in the dorms. Figure 7 charts the occupancy rate at room level and floor level for

\footnotetext{
${ }^{2}$ The stringency of "lockdown" in Singapore was relatively modest compared to those in China, Italy, and Australia, where people could not dwell or travel beyond their immediate neighborhood.
} 


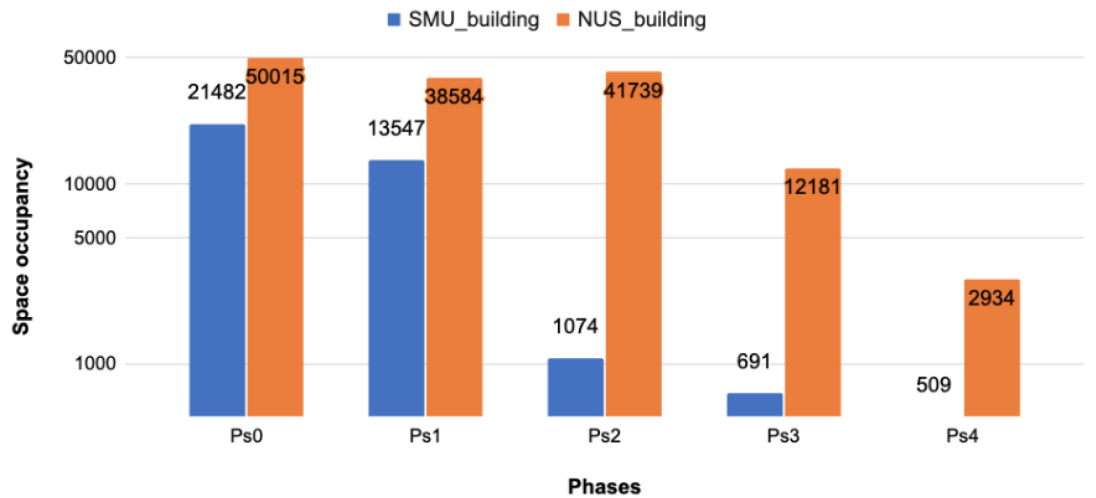

Fig. 6. Building-level occupancy for one school building for each university, SMU (blue) and NUS (orange), plotted from $P_{S} 0$ to $P_{S} 4$.
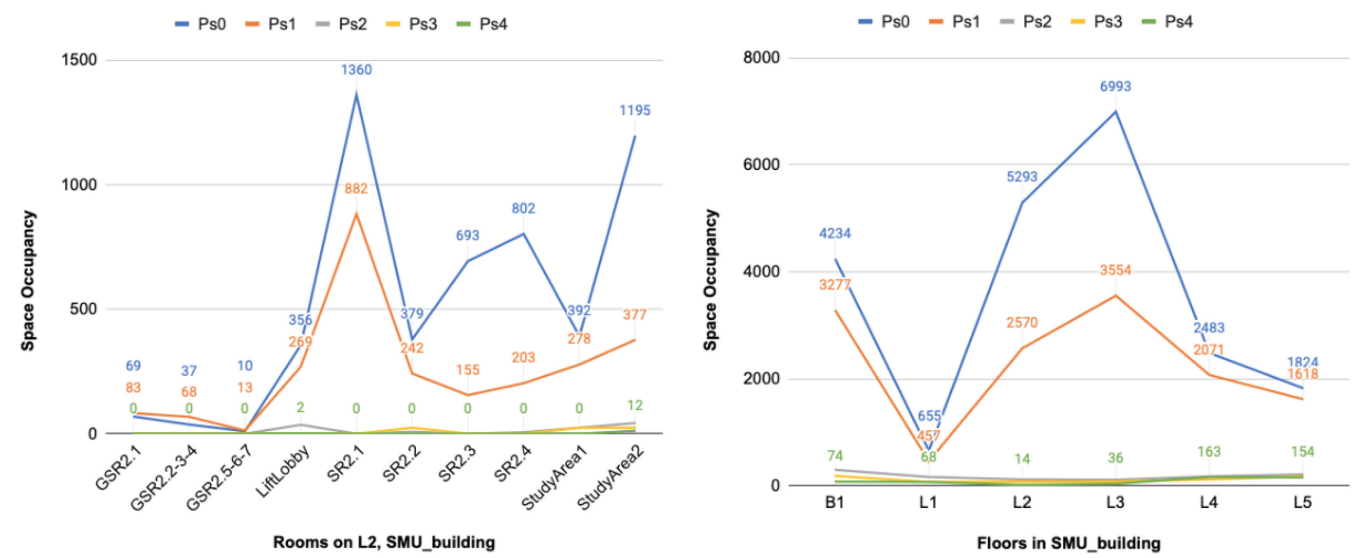

Fig. 7. The percentage change in area-level occupancy within different rooms (left) and levels (right) of $S M U$ buildings, plotted from pre-COVID-19 time $\left(P_{S} 0\right)$ to current time $\left(P_{S} 4\right)$.

$S M U$. A drastic drop in occupancy can be observed as soon as in Phase $1\left(P_{S} 1\right)$ when large classes were shifted to online learning-the space utilization specifically for the seminar rooms (SRs) 2.1 to 2.4 , which are regularly used for holding classes, decreased by more than $50 \%$ on average. Additionally, overall occupancy declined the most for levels 2 and 3, consisting mostly of seminar rooms. While no one was not allowed to work on campus during $P_{S} 4$, clearances were granted and arranged for personnel to bring their workstations home. Note that the occupancy of 12 at study area 2 during $P_{S} 4$ did not last for more than 15 minutes.

To understand this change of occupancy in more detail, Figure 8 shows the percentage change in space occupancy for different types of areas located at three buildings per campus. These areas are dedicated to three activities: recreational, dining, and studying. We considered only the indoor gym area within a building to represent recreational activity, the only common dining hall area within a building to represent dining activity, and multiple study areas on all floors within the library building to represent study activity. The values for $S M U$ are shown in the left figure, while the values for NUS are shown in the right figure. The absolute count for each percentage is listed inside each area (e.g., the absolute count for $S M U_{-}$Food in $P_{S} 4$ is 22 people, making up about $40 \%$ of the total occupancy). 

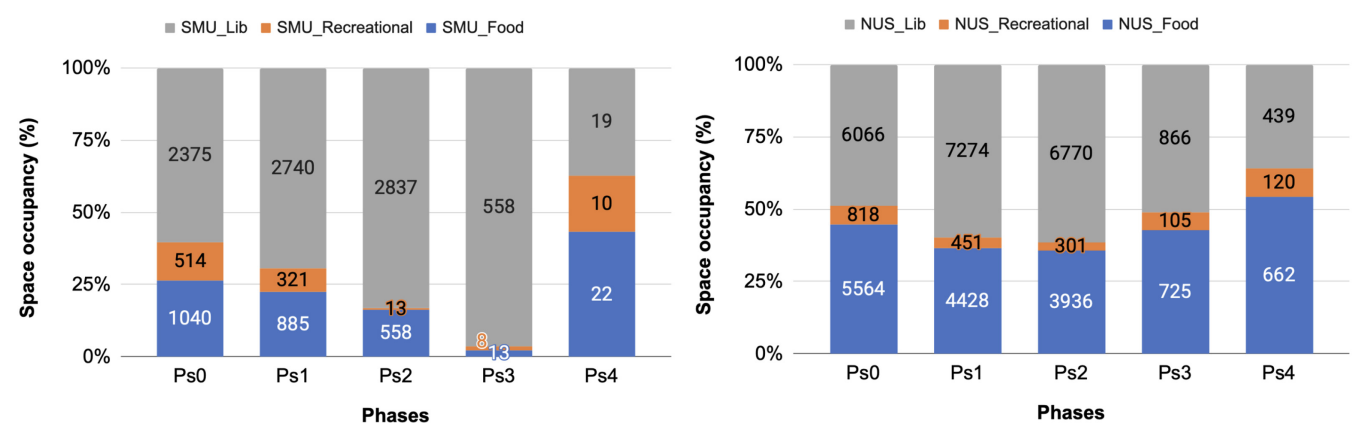

Fig. 8. The percentage change in area-level occupancy within different $S M U$ (left) and NUS (right) buildings, plotted from pre0COVID-19 time $\left(P_{S} 0\right)$ to current time $\left(P_{S} 4\right)$.

There were differences in the space utilization between the two campuses. For example, $S M U$ closed all recreational facilities in $P_{S} 2$ onward, and this is reflected in the noticeable percentage drop. The on-campus dining facilities were also mostly closed from $P_{S} 2$ onward.

As NUS has a large number of students staying on campus in dorms (SMU does not have dorms on campus), even in $P_{S} 4$, the occupancy of recreational spaces was relatively high (and higher percentage-wise than earlier phases). This result is likely because students staying in dorms did not want to stay in their rooms all day long and decided to go out to these recreational spaces (which is technically a violation of the quarantine rules in effect).

Such occurrences raise concerns that these recreational spaces would have larger-than-optimal occupancy densities and undesirable mixing of students from different dorms that would compromise measures designed to contain the spread of infection.

\subsection{Impact of Various Policies on Mobility}

Next, we sought to determine how various social mobility control measures influenced mobility patterns across both universities. First, looking at SMU, Figure 9 charts the average transitions made on a per-building level over a day in each phase, from one building (called SMU_building1) to five other $S M U$ buildings. The transition count indicates the number of times a person moved from $S M U \_$building1 to the indicated building on that day.

The results showed an expected decrease in the overall numbers as each phase was introduced, beginning with the university's decision to conduct online learning for its students at phase $P_{S} 1$. In phase $P_{S} 2, S M U$ introduced full $\mathrm{A} / \mathrm{B}$ schedules where only half the student and staff population could physically be on campus at any one time. This step reduced the overall occupancy (as shown in Figure 6 and decreasing Transition count for $P_{S} 2$ in Figure 9).

The actual mobility rate has also decreased for NUS due to decreased occupancy on campus. However, the mobility rate for each person on campus remained the same-this is understandable as the work required them to visit the same set of buildings they had previously. SMU reduced the campus occupancy to almost 0 in Phase $P_{S} 3$ onward, and this naturally reduced the mobility rate (Figure 9).

We next looked at data from NUS to understand if these changes in mobility patterns were consistent. Figure 10 shows the absolute number of transitions (numbers within each bar) along with the percentage of transitions from one NUS academic building to three other academic buildings. Similar to $S M U$, even though the total occupancy of the campus decreased due to the measures enacted in $P_{S} 2$, the mobility rate (among those staff and students still on campus) remained high until more complete lockdown policies were enacted in $P_{S} 3$ onward. 


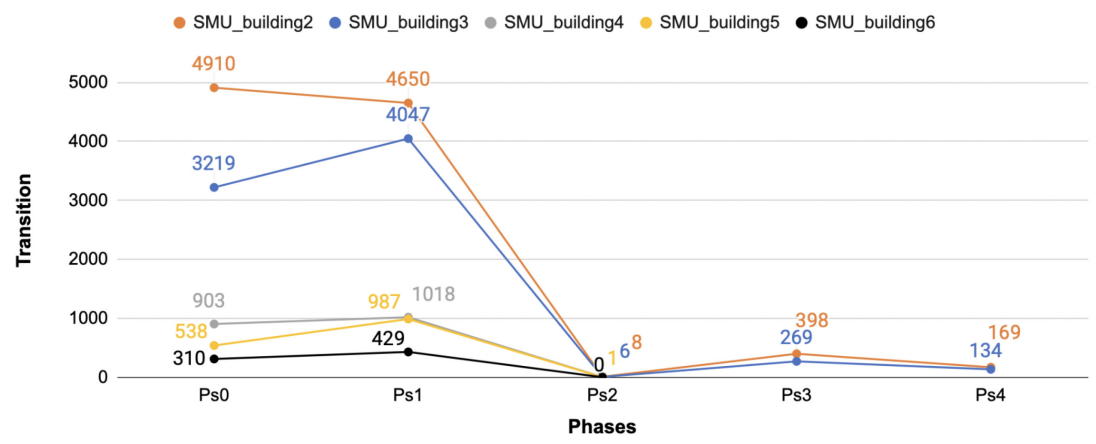

Fig. 9. Device transitions originating from $S M U_{-}$building1 to five other buildings. The patterns of mobility remained the same overall despite a noticeable reduction in the number of transitions over time.

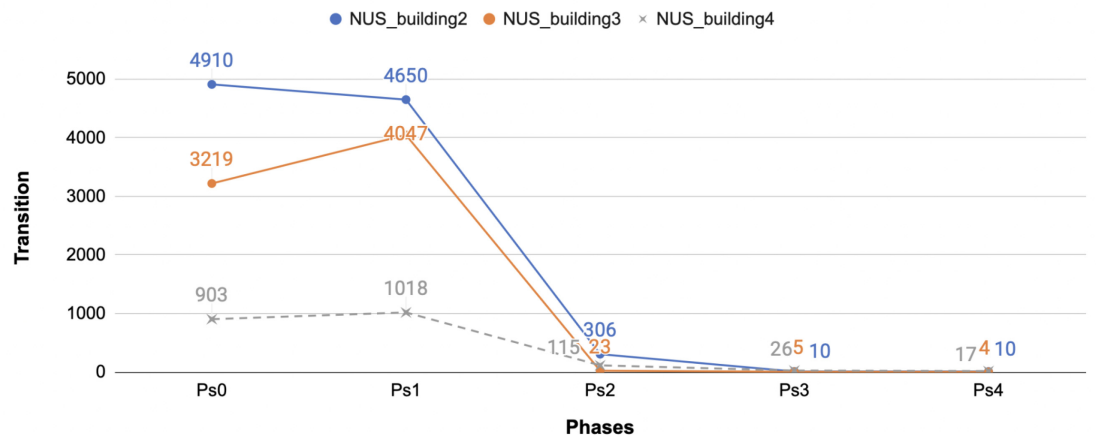

Fig. 10. Device transitions originating from NUS_building 1 to three other academic buildings over different phases. Overall, occupancy rate and mobility rate are evidently less.

\subsection{On-campus Living}

The previous analysis focused on academic buildings. However, NUS, unlike $S M U$, has a significant student population still residing in on-campus dormitories even during the complete lockdown phase $P_{S} 4$. We dug deeper to understand the behavior of students living in these NUS dorms.

Figure 11 plots the daily WiFi activity trend of one dormitory location, NUS_Dorm, over phases $P_{S} 0$ and $P_{S} 4$. Overall, we observed the same daily activity levels (approximately 700 ) for this dorm across all phases-indicating that the dorm population occupancy had not changed between phases. Instead, we observe the full lockdown at $P_{S} 4$ resulting in the reversal of the WiFi activity trend with decreasing connections during the day and active WiFi utilization over the night, indicating user activeness at night.

Our analysis of mobility patterns among students staying in the dorms revealed some interesting findings. In particular, we found that even during lockdown periods $\left(P_{S} 4\right)$, a significant number of people were moving actively across campus-which is technically a violation of the lockdown rules. Figure 12 presents the mobility rate of 200 randomly selected individuals staying in NUS_Dorm to unique areas and AP locations visited within the campus vicinity. We found that their mobility rate increased during $P_{S} 3$ and $P_{S} 4$ compared to $P_{S} 2$. Each occupant was making at least three transitions on average in phase $P_{S} 2$, which doubled to about six transitions in $P_{S} 3$ onward.

While the number of AP locations that individuals' devices were connected to seemed surprisingly high at first glance, these APs remained in neighboring areas of the users' residences. 


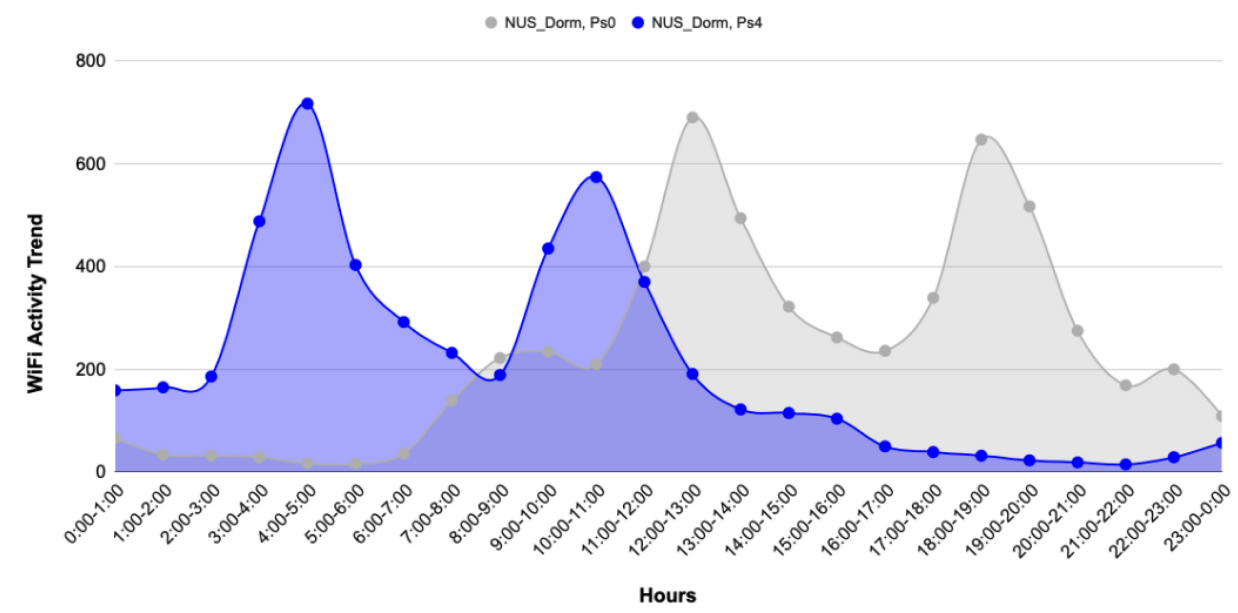

Fig. 11. The hourly WiFi activity trend for NUS_Dorm plotted before $\left(P_{S} 0\right)$ and during full lockdown $\left(P_{S} 4\right)$.

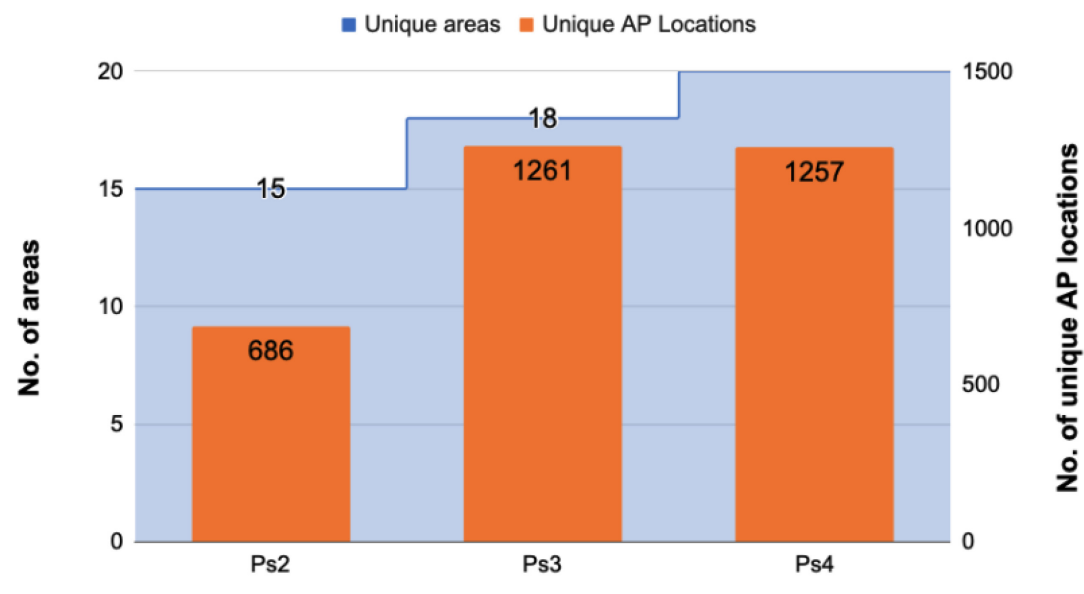

Phases

Fig. 12. Transitions of 200 sampled NUS_Dorm occupants to different (neighborhood) area (e.g., another dorm) as a result of their phones being connected to unique AP locations within NUS.

Specifically, individuals moved between areas in the same dorm to visit the dining, recreational facilities, or bus stops more often (to head to other dining and grocery areas). These were all shorter-length transitions compared to earlier phases-in earlier phases, the transitions had long durations as the individuals were going to academic buildings for coursework reasons.

\subsection{Main Takeaways}

From the analysis of SMU and NUS, the main takeaway we derive is that policies that allow telecommuting and split-team load balancing are excellent for reducing the people density on campus. However, the staff and students that do work on campus are more likely to continue visiting the same set of places they utilized, which can lead to serious issues if an outbreak occurs-as COVID19 can be easily spread to all the other people visiting those areas. Thus, to avoid uncontrolled outbreaks, it may be necessary to limit the mobility of individuals, and the only policy that was 
Table 4. Dates Corresponding to the Safety Measures for COVID-19 in UMASS

\begin{tabular}{lll}
\hline US State Phases & Date Started & \multicolumn{1}{c}{ Safety Measures } \\
\hline Pre COVID-19( $\left.P_{U} 0\right)$ & Feb. 29, 2020 & No policy; business as usual \\
\hline Phase 1 $\left(P_{U} 1\right)$ & Mar. 20, 2020 & Stay-at-home state-wide order [30] \\
& & $\begin{array}{l}\text { No classes at UMASS } \\
\text { Dorms cleared at UMASS except in special cases }\end{array}$ \\
\hline
\end{tabular}

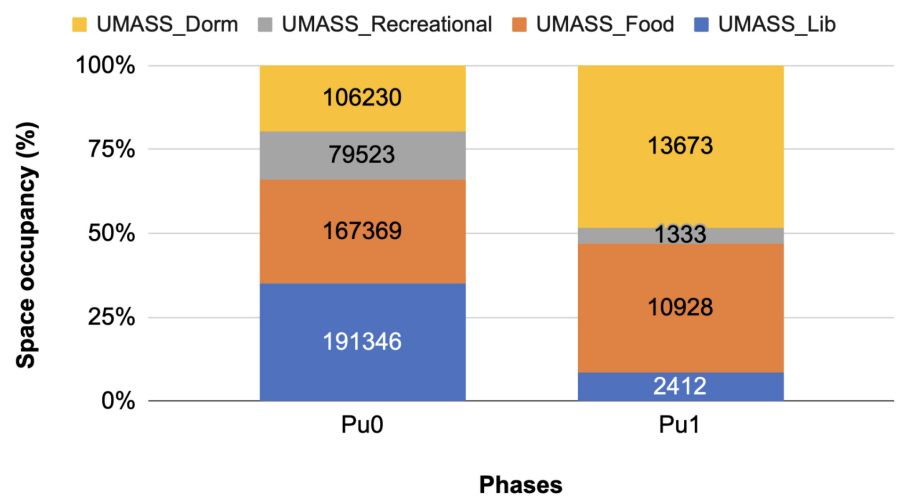

Fig. 13. Space occupancy within UMASS for both $P_{U} 0$ and $P_{U} 1$, broken down by usage type.

successful at this (from the many policies that were tried in Singapore) is a full lockdown where everyone is given stay-at-home orders.

In addition, the mobility analysis of dorm occupants at NUS suggests that reducing mobility will require providing everything occupants need at their premises itself. Otherwise, the mobility rate could go up (even if the actual length of the transitions is shorter in duration) as individuals travel to other places to procure food and other essential items needed during a lockdown.

\section{MOBILITY AND OCCUPANCY ANALYSIS FOR US UNIVERSITY}

Unlike Singapore, the US state that our campus (UMASS) was located in only had a single response-the state went from business as usual to a stay-at-home order with shutdowns of many businesses, as per Table 4 [30]. ${ }^{3}$

We observed similar occupancy trends, compared to SMU and NUS, as UMASS transitioned into a lockdown. Figure 13 plots the total number of unique devices detected for different area types over 10 days for each of the two phases. The areas picked were "Recreational" (e.g., gym), "Dorm" (e.g., on-campus dormitory housing), "Lib" (campus libraries), and "Food" (e.g., food courts). Overall, we observed a more than $90 \%$ decrease in occupancy between phases $P_{U} 0$ (business as usual) and $P_{U} 1$ (full stay-at-home orders). In addition, we observed that the quarantine policies had naturally shifted the occupancy rates, with the dormitories becoming the most occupied locations during $P_{U} 1$ and consequently reducing the occupancy of the previously busy library areas.

We next investigated if similar changes to the mobility rate had occurred due to the quarantine policy. Figure 14 plots the CDF of the number of other places visited by the occupants of one particular UMASS dorm (called UMASS_Dorm) in each phase. We observed that the number of visits had reduced, with the $50^{\text {th }}$ percentile reducing by slightly more than half (about 10 visits in $P_{U} 0$ versus fewer than 5 visits in $P_{U} 1$ ) and the $90^{\text {th }}$ percentile decreasing from about 17 visits to about 9 visits. This reduction in mobility behavior is consistent with [4], which found that the

\footnotetext{
${ }^{3}$ Note: For anonymity reasons, we cite an article listing all the states that have effected a similar policy.
} 


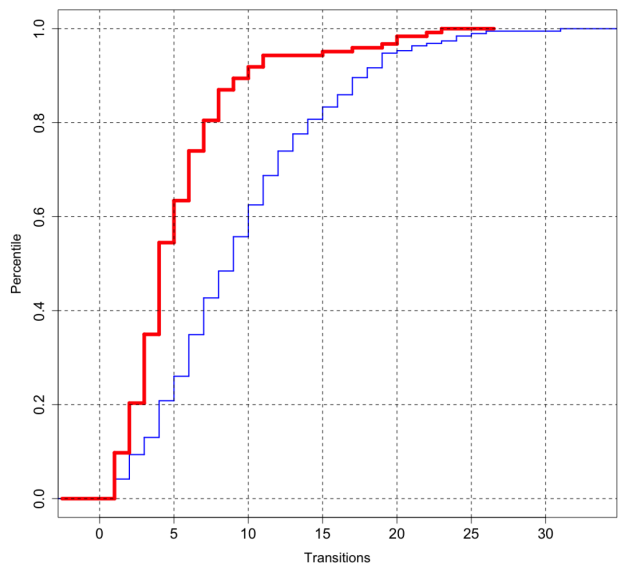

Fig. 14. CDF of unique transition locations made by occupants of UMASS_Dorm, plotted from pre-COVID-19 time $\left(P_{U} 0\right.$, the blue line on the right) to the current quarantine phase $\left(P_{U} 1\right.$, the red line on the left). At least $50 \%$ of the occupants had reduced their transitions from approximately 10 unique locations to 5 .

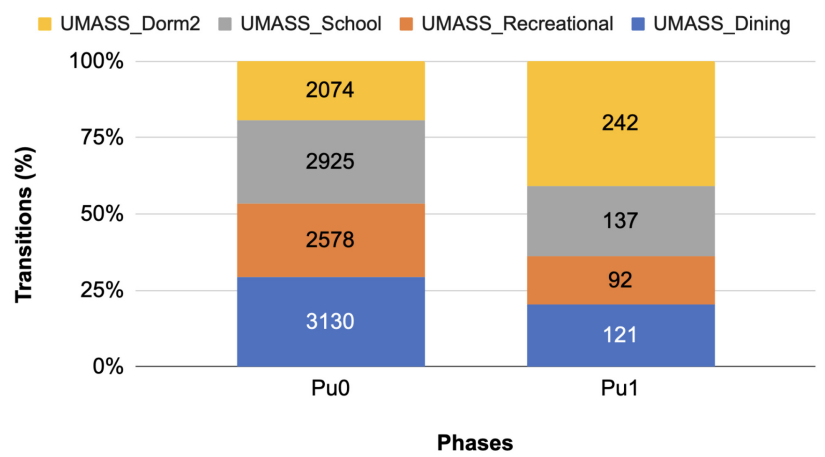

Fig. 15. The percentage change in transitions for different areas made by occupants of UMASS_Dorm, plotted from pre-COVID-19 time $\left(P_{U} 0\right)$ to current time $\left(P_{U} 1\right)$.

change in mobility patterns using cell mobile data reduced by approximately $50 \%$ with state-level policy enforcement.

Figure 15 breaks down these visits by the type of place visited. We observed that most of the visits in $P_{U} 0$ were to dining and recreational locations. However, in $P_{U} 1$, most of the visits were made to another on-campus dorm. We believe this was attributed to the students availing themselves of the dining and recreational facilities as certain previously popular places on campus had been closed in $P_{U} 1$.

\section{POLICY IMPACT}

This section discusses the impact of the policy decisions on disease spreading among the campus community. In particular, we looked at two different modes by which the virus could spread: (1) spreading among the people in the same place as where they are located (we call this vector local spread) and (2) spreading among intercrossing people as they travel to and spend time at places other than the primary location (we call this vector mobility spread). Controlling each of these vectors requires different approaches. 
Controlling local spread requires reducing the density of people in the same location. On the other hand, controlling mobility spread requires reducing the amount of movement outside one's primary location. Reducing the density of people, in general, can reduce mobility spread as well. Note: Both these approaches can apply in both cases except with different intensities. For example, limiting the movement one does in one's primary location can control local spread, but this may not always be practical.

\subsection{Controlling Local Spread across Campus}

From the results presented in Sections 4 and 5, we note that quarantine policies were very effective in removing people from their workplaces. The policies immediately removed one primary source of local spread (i.e., spreading a virus among co-workers).

However, this quarantine policy resulted in higher densities being observed in the student dormitories, as shown in Figure 13 where the occupancy in dorms increased after the initial quarantine measures were imposed. This was "solved" by the universities asking students to vacate the dorms and return home. This reduced the density of the dormitories as shown by our results, but it moved the problem elsewhere. In particular, we believe that many home residences would have seen much higher population densities as a result of these quarantine policies as the entire country (Singapore) and state (in the United States) population was asked to stay at home for extended periods of time. This could make it easier for residents to fall sick if someone in their vicinity had the virus. Indeed, Singapore experienced this first-hand as the second wave of COVID-19 outbreak in Singapore occurred in the dormitories used by foreign workers. The population densities at these dormitories were very high, and the first cases of COVID-19 were reported on April 1, 2020 [38]. This spread grew very fast and resulted in thousands of infections within the dormitories within just a few weeks [36]. Fortunately, the strict quarantine policies, enacted just a few days (on April 7,2020 ) after the first dorm infection (on April 1,2020) when the authorities realized the potential for the spread to grow out of control, ensured that the virus was contained within the dormitories. For example, while the infection rate remains high in the worker dormitories, the number of cases in the rest of Singapore is almost non-existent - on May 15, 2020, 791 new infection cases were discovered in the worker dorms, with just one other case discovered across the rest of Singapore [35]. Thus, while quarantine policies can lead to higher local transmission rates, the significantly reduced mobility stops the virus from spreading beyond the local area.

\subsection{Controlling Mobility Spread}

The second mode of virus propagation is where an infected individual travels to another place and infects someone there. We call this mobility spread. This vector is particularly dangerous as it can allow the virus to spread to formerly safe areas very quickly. Indeed, it was this vector that was responsible for spreading COVID-19 throughout the world-carried by infected individuals traveling between countries.

We see from the results in Section 4 that only a strict quarantine was effective in reducing mobility patterns. In particular, the split team and other approaches used in phases $P_{S} 1$ and $P_{S} 2$ did not have a significant impact on the mobility patterns of individuals (defined as the number of unique places visited by an individual in a day). However, when strict quarantine policies were enacted, starting in $P_{S} 3$ and fully enacted in $P_{S} 4$, we note that the amount of individual mobility has significantly reduced. Also, when people were mobile, they spent significantly lower amounts of time at each place visited.

This data backs up the policy decisions in both Singapore and the US state to enact a strict quarantine as the impact on individual mobility is very clear. Such measures, in turn, dramatically reduce the probability that COVID-19 can spread beyond a local area. However, as stated previously, 
reducing mobility comes at the expense of increasing the population density of homes, dormitories, and other residential areas. Hence, there could be a higher probability of local infections as a result of a strict quarantine (as demonstrated by the worker dormitories outbreak in Singapore).

\section{DISCUSSION}

Our study's objective was to demonstrate that coarse-grained WiFi data can sufficiently reflect the spectrum in crowd change when different COVID-19 control policies were implemented. Here we discuss the implications of our findings.

\subsection{Support Operational Strategies for Universities}

As institutions seek to re-open in the coming semesters, they must fully implement the required COVID-19 protocol and maintain campus operations with as much safety as possible for staff and students. At a higher level, such operational planning encompasses strategies for regulating gatherings in enclosed spaces and tracking infection spread, achievable through monitoring crowd changes using WiFi sensing. Specifically, as shown in Figures 3 and 4, reports on actual space utilization can emphasize over-utilized areas and buildings' floor-by-floor foot traffic. Monitoring mobility as per Figure 5 can emphasize possible transmission routes within and among buildings in the campus vicinity. As a whole, monitoring occupancy and mobility can aid institutions' backtrack path and areas to focus on disinfecting compromised sites. Other strategies relate to regulating restrictions for external parties and planning for emergency evacuation. The ability to distinguish user types on the WiFi network, as shown in Figure 2, can inform institutions of overcapacity visitors. Monitoring total building capacity remains relevant in reducing the risk of occupant exposure to infection spread in shared open spaces, for example, establishing one-way safe travels within buildings in the event of an emergency evacuation.

\subsection{Practical Application for Institutional Crowd Controls}

We have since developed an open sourced monitoring platform that allows institutions to set occupancy parameters based on their own risk assessment for infection spread and mobilize contact tracing within our university campuses: https://github.com/umassos/elastic-wifitrace.

Figure 16 is a dashboard visualization of users at UMASS, driven from their WiFi network device activity. University administrators can select a building and its respective floor to narrow down monitoring to a specific area (e.g., an open study area); see Filter 1 and 2. As explained in Section 3.3, we used the number of unique MAC addresses seen by our WiFi network (devices) to calculate occupancy percentages (Pane 3 and 4). The ability to refine our monitoring parameters and results from per building to areas per floor can quickly help administrators determine areas that may violate zone restrictions. For example, as per Panes 5 and 6, the occupancy heatmaps for each area within the building show a relatively high occupancy rate (in orange) for five areas throughout the day. Note: Each area is represented as a row in the y-axis, while the $\mathrm{x}$-axis denotes the time of day.

From an operational perspective, institutions can make more informed decisions to actively revise the restricted capacity based on the severity of an outbreak or official safety compliance guidelines. Alerts on over-utilized spaces help administrators appropriately deploy officers on the ground to manage the crowd. Attention to under-utilized buildings can also be an opportunity for scheduling events in separate physical locations while complying with building occupancy limitations.

\subsection{Implementation of Privacy Safeguards}

Our goal is toward establishing a non-privacy-invasive modality to monitor indoor traffic and implement disease control policies. Recall in Section 2.4 that we described our privacy safeguards 


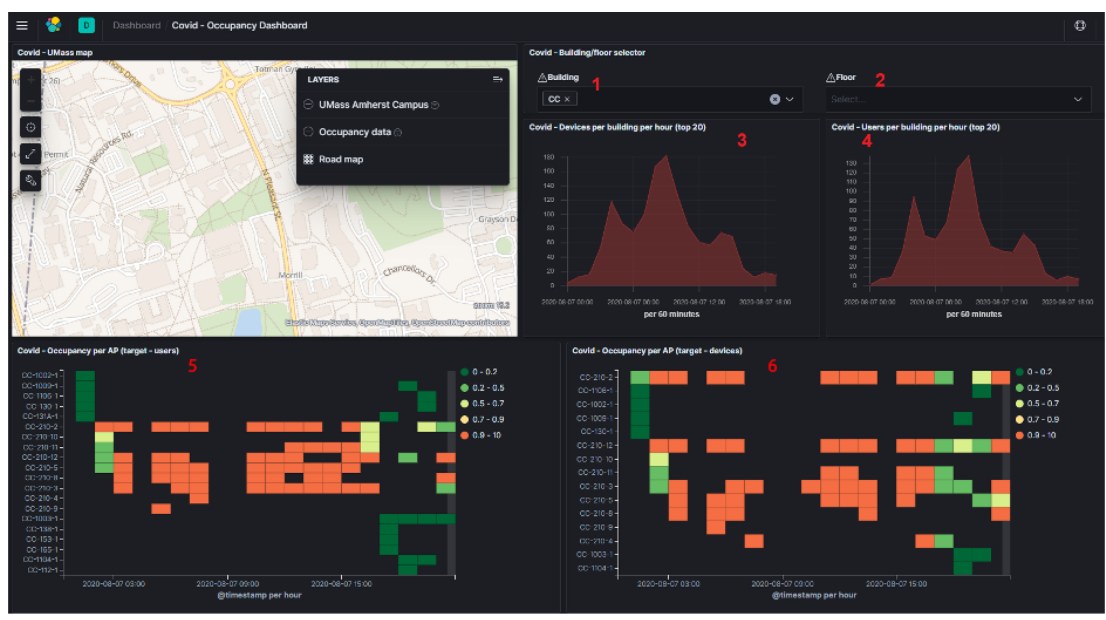

Fig. 16. A dashboard visualization of occupancy rate in areas per floor of a building.

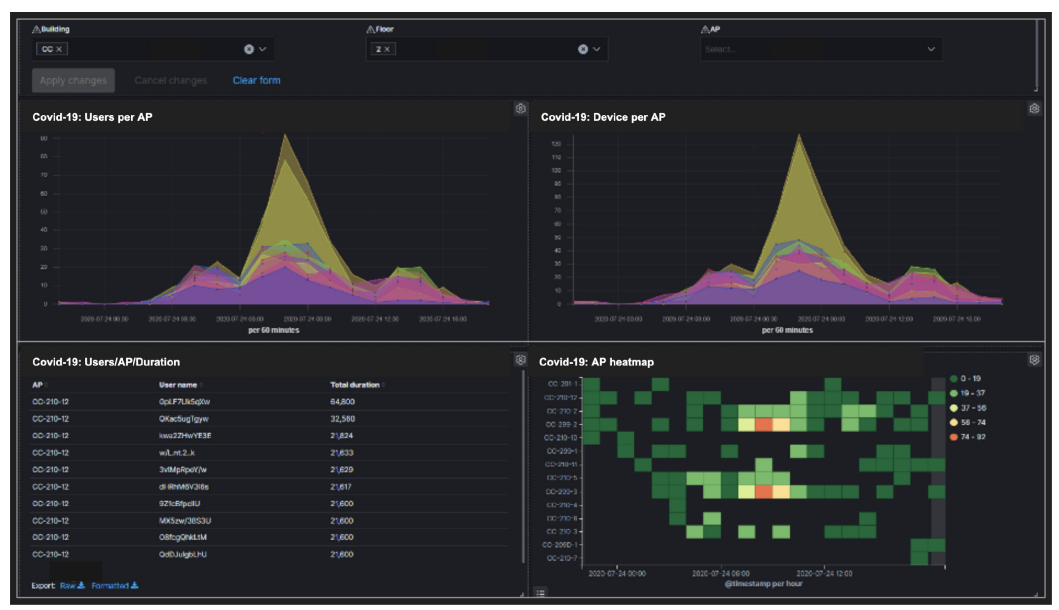

Fig. 17. User dashboard displaying all relevant information of occupancy density based on a target location. Left bottom pane lists anonymized occupants.

in the event an area is determined to be at high risk of COVID-19 spread, and contract tracing may need to be performed. As shown in Figure 17 (bottom left pane), we have implemented Elastic WiFiTrace to utilize anonymized WiFi data for measuring occupancy. The mechanism of deanonymizing this information is recommended for university administrators to determine occupants at risk of potential exposure. We suggest that a standard mandatory notice and consent provision is provided to users of the campus WiFi network to allow for WiFi-based contact tracing. Upon consent, a user preemptively authorizes administrators to access key information, particularly their MAC ID and username, to initiate a manual contact tracing procedure.

Overall, we believe passive WiFi sensing is a promising technique to pursue COVID-19 response on a larger scale. We are already providing updates at $S M U$ to the campus facilities managers and the deans of students at NUS regarding the occupancy and mobility levels across the buildings and dorms at each campus. 


\section{CONCLUSION \& FUTURE WORK}

Amid this pandemic, we can anticipate increasingly vulnerable situations to arise as individuals congregate in groups. This article presented results from two campuses in Singapore and one in the Northeastern portion of the United States, demonstrating how WiFi network information alone could reveal occupancy and mobility spectrum changing with different containment policies. Indeed, augmenting $\mathrm{WiFi}$ data can assist institutions with managing safety compliance as we get through the crisis. Our open source COVID-19 monitoring tool is available: https://github.com/umassos/elastic-wifitrace.

This study is not without limitations. First, the data was only collected from WiFi-enabled devices that were associated with the campus WiFi networks; this can result in under-counting if individuals do not enable or carry a WiFi device or are not connected to the campus network. Second, WiFi sensing does not produce fine-grained spatial measures, including occupancy in small rooms that share WiFi APs and inter-user distances. Thus, the modality cannot directly determine individuals who stand too close to an infected person and regard them as at risk of exposure. Instead, it identifies occupants' degree of risk from being in an exposed area (e.g., an hour-long meeting in a conference room or a lecture classroom), which remains relevant to institutions conducting follow-up manual contact tracing. Third and finally, our data comes from two countries with contrasting COVID-19 policies. While we have observed similar results, local factors may prevent specific results from applying to other regions. As COVID-19 continues to spread, our efforts progress toward deeper and broader analyses addressing these shortfalls.

\section{REFERENCES}

[1] ABC News. 2020. States Look to Closed Hospitals and College Dorms to Meet Coronavirus Demands. https://abcnews.go. com/Politics/states-closed-hospitals-college-dorms-meet-coronavirus-demands/story?id=69683447.

[2] Aruba Networks. 2020. IT Analytics for Operational Intelligence. https://www.arubanetworks.com/products/locationservices/analytics/.

[3] Roshan Ayyalasomayajula, Deepak Vasisht, and Dinesh Bharadia. 2018. BLoc: CSI-based accurate localization for BLE tags. In Proceedings of the 14th International Conference on Emerging Networking EXperiments and Technologies. 126-138.

[4] Hamada S. Badr, Hongru Du, Maximilian Marshall, Ensheng Dong, Marietta M. Squire, and Lauren M. Gardner. 2020. Association between mobility patterns and COVID-19 transmission in the USA: A mathematical modelling study. Lancet Infectious Diseases 20, 11 (2020), 1247-1254.

[5] Ahmed Barnawi, Prateek Chhikara, Rajkumar Tekchandani, Neeraj Kumar, and Bander Alzahrani. 2021. Artificial intelligence-enabled internet of things-based system for COVID-19 screening using aerial thermal imaging. Future Generation Computer Systems 124 (2021), 119-132.

[6] Center for Systems Science and Engineering (CSSE). 2020. COVID-19 Dashboard. https://gisanddata.maps.arcgis.com/ apps/opsdashboard/index.html\#/bda7594740fd40299423467b48e9ecf6.

[7] Centers for Disease Control and Prevention (CDC). 2020. Considerations for institutions of higher education. Retrieved June 25, 2020).

[8] Centers for Disease Control and Prevention (CDC). 2022. Operational Guidance for K-12 Schools and Early Care and Education Programs to Support Safe In-Person Learning. https://www.cdc.gov/coronavirus/2019-ncov/community/ schools-childcare/k-12-childcareguidance.html.

[9] Cisco. 2019. Higher Education Institutions Prepare for Wi-Fi 6. https://meraki.cisco.com/blog/2019/05/higher-educationinstitutions-prepare-for-wi-fi-6/.

[10] Cisco. 2020. Cisco DNA Spaces. https://www.cisco.com/c/en/us/solutions/enterprise-networks/dna-spaces/index. html.

[11] CORONATRACKER.com. 2020. COVID-19 Corona Tracker. https://www.coronatracker.com/.

[12] Helen Crompton and Diane Burke. 2018. The use of mobile learning in higher education: A systematic review. Computers \& Education 123 (2018), 53-64.

[13] Aaqib Bashir Dar, Auqib Hamid Lone, Saniya Zahoor, Afshan Amin Khan, and Roohie Naaz. 2020. Applicability of mobile contact tracing in fighting pandemic (Covid-19): Issues, challenges and solutions. Computer Science Review 38 (2020), 100307. 
[14] Dorine C. Duives, Tim van Oijen, and Serge P. Hoogendoorn. 2020. Enhancing crowd monitoring system functionality through data fusion: Estimating flow rate from Wi-Fi traces and automated counting system data. Sensors 20, 21 (2020), 6032 .

[15] Singapore Economic Development Board. 2020. Safe Management Measures at the Workplace. https://www.edb.gov.sg/ en/how-we-help/supporting-you-through-covid-19.html.

[16] Government of Singapore. 2020. SafeEntry National Digital Check-in System. https://www.safeentry.gov.sg.

[17] Government of Singapore. 2020. TraceTogether. https://www.tracetogether.gov.sg.

[18] Bin Guo, Zhu Wang, Zhiwen Yu, Yu Wang, Neil Y. Yen, Runhe Huang, and Xingshe Zhou. 2015. Mobile crowd sensing and computing: The review of an emerging human-powered sensing paradigm. ACM Computing Surveys (CSUR) 48 , 1 (2015), 1-31.

[19] Gabriella M. Harari, Nicholas D. Lane, Rui Wang, Benjamin S. Crosier, Andrew T. Campbell, and Samuel D. Gosling. 2016. Using smartphones to collect behavioral data in psychological science: Opportunities, practical considerations, and challenges. Perspectives on Psychological Science 11, 6 (2016), 838-854.

[20] Illhoe Hwang and Young Jae Jang. 2017. Process mining to discover shoppers' pathways at a fashion retail store using a WiFi-base indoor positioning system. IEEE Transactions on Automation Science and Engineering 14, 4 (2017), 1786-1792.

[21] Immigration and Checkpoints Authority, Singapore. 2020. Travellers Arriving In Singapore Will Receive Advance Notification Of Stay-Home Notice Requirements. https://www.ica.gov.sg/news-and-publications/media-releases/mediarelease/travellers-arriving-in-singapore-will-receive-advance-notification-of-stay-home-notice-requirements.

[22] Infocomm Media Development Authority (IMDA). 2020. Digital Solutions for Safe Reopening. https://www.imda.gov. sg/programme-listing/smes-go-digital/Digital-Solutions-For-Safe-Reopening.

[23] International Association of Public Transport, UITP. 2020. New EU-funded Application CO-APS Aims to Reduce Spread of COVID-19 in Public Transport. https://www.uitp.org/news/new-eu-funded-application-co-aps-aims-to-reducespread-of-covid-19-in-public-transport/.

[24] International Labour Organization. 2020. Country Policy Responses. https://www.ilo.org/global/topics/coronavirus/ country-responses/lang--en/index.htm.

[25] Samvit Jain, Ganesh Ananthanarayanan, Junchen Jiang, Yuanchao Shu, and Joseph Gonzalez. 2019. Scaling video analytics systems to large camera deployments. In Proceedings of the 20th International Workshop on Mobile Computing Systems and Applications. 9-14.

[26] Kasthuri Jayarajah, Rajesh Krishna Balan, Meera Radhakrishnan, Archan Misra, and Youngki Lee. 2016. Livelabs: Building in-situ mobile sensing \& behavioural experimentation testbeds. In Proceedings of the 14th Annual International Conference on Mobile Systems, Applications, and Services. 1-15.

[27] Kasthuri Jayarajah, Youngki Lee, Archan Misra, and Rajesh Krishna Balan. 2015. Need accurate user behaviour? pay attention to groups! In Proceedings of the 2015 ACM International foint Conference on Pervasive and Ubiquitous Computing. 855-866.

[28] Karen Kent and Murugiah Souppaya. 2006. Guide to Computer Security Log Management: Recommendations of the National Institute of Standards and Technology. US Department of Commerce, Technology Administration, National Institute of 2006.

[29] Adarsh Kumar, Kriti Sharma, Harvinder Singh, Sagar Gupta Naugriya, Sukhpal Singh Gill, and Rajkumar Buyya. 2021. A drone-based networked system and methods for combating coronavirus disease (COVID-19) pandemic. Future Generation Computer Systems 115 (2021), 1-19.

[30] Alicia Lee. 2020. These States Have Implemented Stay-at-home Orders. Here's What That Means for You. https://edition. cnn.com/2020/03/23/us/coronavirus-which-states-stay-at-home-order-trnd/index.html.

[31] David Lee and Jaehong Lee. 2020. Testing on the move South Korea's rapid response to the COVID-19 pandemic. Transportation Research Interdisciplinary Perspectives 5 (2020), 100111.

[32] M. Lewis. 2020. COVID-19 outbreak among college students after a spring break trip to Mexico-Austin, Texas, March 26-April 5, 2020. MMWR. Morbidity and mortality weekly report, 69.

[33] Xiaoxuan Lu, Hongkai Wen, Han Zou, Hao Jiang, Lihua Xie, and Niki Trigoni. 2016. Robust occupancy inference with commodity WiFi. In 2016 IEEE 12th International Conference on Wireless and Mobile Computing, Networking and Communications (WiMob'16). IEEE, 1-8.

[34] Huadong Ma, Dong Zhao, and Peiyan Yuan. 2014. Opportunities in mobile crowd sensing. IEEE Communications Magazine 52, 8 (2014), 29-35.

[35] Ministry of Health, Singapore. 2020. 1,275 More Cases Discharged, 793 New Cases of COVID-19 Infection Confirmed. https://www.moh.gov.sg/news-highlights/details/1-275-more-cases-discharged-793-new-cases-of-covid-19infection-confirmed.

[36] Ministry of Health, Singapore. 2020. 1,426 New Cases of COVID-19 Infection. https://www.moh.gov.sg/news-highlights/ details/1-426-new-cases-of-covid-19-infection. 
[37] Ministry of Health, Singapore. 2020. Circuit Breaker to Minimise Further Spread of COVID-19. https://www.moh.gov. $\mathrm{sg} /$ news-highlights/details/circuit-breaker-to-minimise-further-spread-of-covid-19.

[38] Ministry of Health, Singapore. 2020. Five More Cases Discharged; 74 New Cases of COVID-19 Infection Confirmed. https://www.moh.gov.sg/news-highlights/details/five-more-cases-discharged-74-new-cases-of-covid-19-infectionconfirmed.

[39] Ministry of Health, Singapore. 2020. Mid-year Holidays Brought Forward as Schools Adjust Academic Calendar; Institutes of Higher Learning to Extend Home-based Learning. https://www.moe.gov.sg/news/press-releases/mid-yearholidays-brought-forward-as-schools-adjust-academic-calendar-institutes-of-higher-learning-to-extend-homebased-learning.

[40] Ministry of Health, Singapore. 2020. Risk Assessment Raised to DORSCON Orange. https://www.moh.gov.sg/newshighlights/details/risk-assessment-raised-to-dorscon-orange.

[41] Ministry of Health, Singapore. 2020. Schools and Institutes of Higher Learning to Shift to Full Home-based Learning; Preschools and Student Care Centres to Suspend General Services. https://www.moe.gov.sg/news/press-releases/schoolsand-institutes-of-higher-learning-to-shift-to-full-home-based-learning-preschools-and-student-care-centres-tosuspend-general-services.

[42] Ministry of Manpower, Singapore. 2020. Mandatory Stay-home Notice for Work Pass Holders with Travel History to Mainland China. https:/www.mom.gov.sg/newsroom/press-releases/2020/0214-mandatory-shn-for-wph-with-travelhistory-to-china.

[43] Occupational Safety and Health Administration (OSHA). 2020. Regulations. https://www.osha.gov/coronavirus/ standards.

[44] John R. B. Palmer, Thomas J. Espenshade, Frederic Bartumeus, Chang Y. Chung, Necati Ercan Ozgencil, and Kathleen Li. 2013. New approaches to human mobility: Using mobile phones for demographic research. Demography 50, 3 (2013), 1105-1128.

[45] Savvas Papaioannou, Andrew Markham, and Niki Trigoni. 2016. Tracking people in highly dynamic industrial environments. IEEE Transactions on Mobile Computing 16, 8 (2016), 2351-2365.

[46] Shin Young Park, Young-Man Kim, Seonju Yi, Sangeun Lee, Baeg-Ju Na, Chang Bo Kim, Jung-Il Kim, Hea Sook Kim, Young Bok Kim, Yoojin Park, et al. 2020. Coronavirus disease outbreak in call center, South Korea. Emerging Infectious Diseases 26, 8 (2020), 1666.

[47] Rijurekha Sen, Youngki Lee, Kasthuri Jayarajah, Archan Misra, and Rajesh Krishna Balan. 2014. Grumon: Fast and accurate group monitoring for heterogeneous urban spaces. In Proceedings of the 12th ACM Conference on Embedded Network Sensor Systems. 46-60.

[48] Mohammad Shorfuzzaman, M. Shamim Hossain, and Mohammed F. Alhamid. 2021. Towards the sustainable development of smart cities through mass video surveillance: A response to the COVID-19 pandemic. Sustainable Cities and Society 64 (2021), 102582.

[49] Vital Surveillances. 2020. The epidemiological characteristics of an outbreak of 2019 novel coronavirus diseases (COVID-19)-China, 2020. China CDC Weekly 2, 8 (2020), 113-122.

[50] Amelia Teng. 2020. NUS to House Recovering Covid-19 Patients at Prince George's Park Residences. https://www. straitstimes.com/singapore/coronavirus-nus-to-house-patients-recovering-from-covid-19-at-prince-georges-park.

[51] The New York Times (NYT). 2020. What We Know About Coronavirus Cases on Campus. https://www.nytimes.com/ interactive/2020/07/28/us/covid-19-colleges-universities.html.

[52] The New York Times (NYT). 2021. How Singapore Has Kept the Coronavirus Off Campus. https://www.nytimes.com/ 2021/01/09/world/asia/singapore-coronavirus-universities.html.

[53] Amee Trivedi, Camellia Zakaria, Rajesh Balan, Ann Becker, George Corey, and Prashant Shenoy. 2021. WiFiTrace: Network-based contact tracing for infectious diseases using passive WiFi sensing. Proceedings of the ACM on Interactive, Mobile, Wearable and Ubiquitous Technologies 5, 1 (2021), 1-26.

[54] Rui Wang, Fanglin Chen, Zhenyu Chen, Tianxing Li, Gabriella Harari, Stefanie Tignor, Xia Zhou, Dror Ben-Zeev, and Andrew T. Campbell. 2014. StudentLife: Assessing mental health, academic performance and behavioral trends of college students using smartphones. In Proceedings of the 2014 ACM International foint Conference on Pervasive and Ubiquitous Computing. 3-14.

[55] World Health Organization (WHO). 2020. Coronavirus Disease (COVID-2019) Situation Reports. https://www.who.int/ emergencies/diseases/novel-coronavirus-2019/situation-reports.

[56] Worldometer. 2020. COVID-19 Coronovirus Pandemic. https://www.worldometers.info/coronavirus/.

[57] Huatao Xu, Dong Wang, Run Zhao, and Qian Zhang. 2019. AdaRF: Adaptive RFID-based indoor localization using deep learning enhanced holography. Proceedings of the ACM on Interactive, Mobile, Wearable and Ubiquitous Technologies 3 , 3 (2019), 1-22.

[58] Jason Chin-Huat Yap, Ian Yi Han Ang, Sharon Hui Xuan Tan, I. Jacinta, Chen Pei, Ruth Frances Lewis, Yang Qian, Rowena Kah Sin Yap, Bob Xian Yi Ng, and Hao Yi Tan. 2020. COVID-19 Science Report: Exit Strategies \& Scenarios. Technical Report. National University of Singapore. 
[59] Camellia Zakaria, Rajesh Balan, and Youngki Lee. 2019. StressMon: Scalable detection of perceived stress and depression using passive sensing of changes in work routines and group interactions. Proceedings of the ACM on HumanComputer Interaction 3, CSCW (2019), 1-29.

[60] Cheng Zhang, Yunze Pan, Yunqi Zhang, Adam C. Champion, Zhaohui Shen, Dong Xuan, Zhiqiang Lin, and Ness B. Shroff. 2021. WLAN-log-based superspreader detection in the COVID-19 pandemic. High-Confidence Computing 1, 1 (2021), 100005.

[61] Yang Zhen, Masato Sugasaki, Yoshihiro Kawahara, Kota Tsubouchi, Matthew Ishige, and Masamichi Shimosaka. 2021. AI-BPO: Adaptive incremental BLE beacon placement optimization for crowd density monitoring applications. In Proceedings of the 29th International Conference on Advances in Geographic Information Systems. 301-304.

Received April 2021; revised January 2022; accepted February 2022 